Research Article

\title{
Tempered Mittag-Leffler Stability of Tempered Fractional Dynamical Systems
}

\author{
Jingwei Deng $(\mathbb{D}$, Weiyuan Ma $(\mathbb{D}$, Kaiying Deng $(\mathbb{D}$, and Yingxing Li \\ School of Mathematics and Computer Science, Northwest Minzu University, Lanzhou 730030, China \\ Correspondence should be addressed to Weiyuan Ma; mwy2004@126.com
}

Received 11 February 2020; Revised 1 May 2020; Accepted 14 May 2020; Published 29 May 2020

Guest Editor: Cuimei Jiang

Copyright (C) 2020 Jingwei Deng et al. This is an open access article distributed under the Creative Commons Attribution License, which permits unrestricted use, distribution, and reproduction in any medium, provided the original work is properly cited.

\begin{abstract}
Due to finite lifespan of the particles or boundedness of the physical space, tempered fractional calculus seems to be a more reasonable physical choice. Stability is a central issue for the tempered fractional system. This paper focuses on the tempered Mittag-Leffler stability for tempered fractional systems, being much different from the ones for pure fractional case. Some new lemmas for tempered fractional Caputo or Riemann-Liouville derivatives are established. Besides, tempered fractional comparison principle and extended Lyapunov direct method are used to construct stability for tempered fractional system. Finally, two examples are presented to illustrate the effectiveness of theoretical results.
\end{abstract}

\section{Introduction}

Fractional derivatives were first proposed by Leibnitz soon after the more familiar classic integer order derivatives. In recent decades, the study of fractional differential systems has attracted wide attention. Compared with the classical calculus, fractional calculus can better characterize memory and hereditary properties of processes and materials. They are now used to model the dynamical evolution in the fields of physics, chemistry, biology, and so on. Fractional calculus can be most easily understood in terms of probability. The relationships among random walks, Brownian motion, and diffusion processes were given in [1]. It is more reasonable to replace classic derivatives by fractional analogues in the diffusion equation [2].

Fractional calculus involves the operation of convolution with a power law function. Multiplying by an exponential factor results in tempered fractional derivatives and integrals [3], this exponential tempering has many merits both in mathematical and practical. A truncated Lévy flight was investigated to capture the natural cutoff in real physical systems [4]. Without a sharp cutoff, the tempered Lévy flight was studied as a smoother alternative [5]. Cartea and del-CastilloNegrete [6] explored the tempered fractional diffusion equation by the tempered Lévy flight. In finance, the tempered stable process models describe price fluctuations with semiheavy tails
[7-10]. Tempered fractional time derivatives can be also found in geophysics [11-13], Brownian motion [14], and so on.

As in classical calculus, stability analysis is still one of the most important tasks in fractional differential system [15-20]. It is a basic feature in fractional physical and biological systems, such as Duffing oscillator [21], neural networks [22-24], and predator-prey models [25]. At present, Lyapunov method has been applied to analyze Mittag-Leffler stability of different fractional systems [26-30]. Li et al. [26, 27] obtained a series of conclusions on the Mittag-Leffler stable for nonlinear fractional equations. In [28], Mittag-Leffler stability of multiple equilibrium points of fractional recurrent neural networks was considered. In [29], a convex and positive definite function was used to analyze Mittag-Leffler stable for fractional system. In [30], the authors presented the Lyapunov stability analysis for fractional nonlinear systems with impulses.

As far as we know, no paper has discussed stability analysis for tempered fractional system. Motivated by this, we think it is very necessary and meaningful to study Mittag-Leffler stability of tempered fractional dynamical systems both in theoretical research and practical application. Because tempered fractional operators combine with nonlocal, weak singularity, and exponential factors [31-33], it has many differences to fractional case in stability analysis. In this paper, tempered Mittag-Leffler 
stability is first proposed. It is a more appropriate concept for tempered fractional system. Tempered comparison principle and some inequalities are given for tempered fractional calculus or systems. Then, sufficient conditions for tempered Mittag-Leffler stability are provided and verified by the Lyapunov method. Finally, the theoretical results are applied to some examples.

This paper is organized as follows. In Section 2, some necessary definitions and lemmas are prepared. Section 3 mainly discusses the sufficient criterions ensuring Mittag-Leffler stability of the tempered fractional systems. In Section 4, two examples are presented to show the effectiveness of theoretical results. We conclude the paper with some discussions in Section 5.

\section{Preliminaries}

Tempered fractional calculus plays an important role in different fields $[34,35]$. In practical application, many different tempered fractional derivatives are proposed, such as Caputo, Riemmann-Liouville, and Riesz. Some definitions and lemmas are stated below, which will be used later.

Definition 1 (see [13]). The tempered fractional integral of order $\alpha>0$ and tempered parameter $\lambda \geq 0$ is defined as

$$
{ }_{a} I_{t}^{\alpha, \lambda} x(t)=\frac{1}{\Gamma(\alpha)} \int_{a}^{t} e^{-\lambda(t-s)}(t-s)^{\alpha-1} x(s) \mathrm{d} s,
$$

where $\Gamma$ presents the Euler gamma function.

Definition 2 (see [3]). The tempered fractional Caputo derivative of tempered parameter $\lambda \geq 0$ is defined as

$$
\begin{aligned}
{ }_{a}^{C} D_{t}^{\alpha, \lambda} x(t):=e^{-\lambda t C} D_{t}^{\alpha}\left(e^{\lambda t} u(t)\right)= & \frac{e^{-\lambda t}}{\Gamma(n-\alpha)} \int_{a}^{t} \frac{1}{(t-s)^{\alpha-n+1}} \\
& \cdot \frac{\mathrm{d}^{n}\left(e^{\lambda s} x(s)\right)}{\mathrm{d} s^{n}} \mathrm{~d} s
\end{aligned}
$$

where $n-1 \leq \alpha<n, n \in \mathbb{N}$, and ${ }_{a}^{C} D_{t}^{\alpha}$ is the Caputo fractional derivative.

Definition 3 (see [9]). The tempered fractional Riemann-Liouville derivative of tempered parameter $\lambda \geq 0$ is defined as

$$
{ }_{a} D_{t}^{\alpha, \lambda} u(t):=e^{-\lambda t}{ }_{a} D_{t}^{\alpha}\left(e^{\lambda t} u(t)\right)=\frac{e^{-\lambda t}}{\Gamma(n-\alpha)} \frac{\mathrm{d}^{n}}{\mathrm{~d} t^{n}} \int_{a}^{t} \frac{e^{\lambda s} u(s)}{(t-s)^{\alpha-n+1}} \mathrm{~d} s,
$$

where $n-1 \leq \alpha<n, n \in \mathbb{N}$, and ${ }_{a} D_{t}^{\alpha}$ is the Riemann-Liouville fractional derivative operator.

In order to study the stability of tempered fractional systems, several lemmas are needed.

Lemma 1 (see [36]). Let $0<\alpha<1, \lambda \geq 0$ and $t \geq a$, then

$$
{ }_{a}^{C} D_{t}^{\alpha, \lambda}(x(t))={ }_{a} D_{t}^{\alpha, \lambda}(x(t))-\frac{e^{-\lambda t}(t-a)^{-\alpha}}{\Gamma(1-\alpha)}\left[e^{\lambda t} x(t)_{t=a}\right] .
$$

Lemma 2 (see [36]). Let $0<\alpha<1, \lambda \geq 0$, then

$$
\begin{aligned}
& \text { (i) }{ }_{a} I_{t}^{\alpha, \lambda}\left[{ }_{a}^{C} D_{t}^{\alpha, \lambda} x(t)\right]=x(t)-e^{-\lambda(t-a)} x(a), \quad{ }_{a}^{C} D_{t}^{\alpha, \lambda}\left[{ }_{a} I_{t}^{\alpha, \lambda}\right. \\
& x(t)]=x(t) \\
& \text { (ii) }{ }_{a} I_{t}^{\alpha, \lambda}\left[{ }_{a} D_{t}^{\alpha, \lambda} x(t)\right]=x(t)-\left(e^{-\lambda t}(t-a)^{\alpha-1} / \Gamma(\alpha)\right) \\
& \\
& {\left[\left.{ }_{a} D_{t}^{\alpha, \lambda}\left(e^{\lambda t} u(t)\right)\right|_{t=a}\right] \text { and }{ }_{a} D_{t}^{\alpha, \lambda}\left[{ }_{a} I_{t}^{\alpha, \lambda} x(t)\right]=x(t)}
\end{aligned}
$$

Lemma 3 (see [36]). The Laplace transform of tempered fractional integral and Caputo derivative (2) are given as

(i) $\mathscr{L}\left\{{ }_{0} I_{t}^{\alpha, \lambda}(x(t))\right\}=(\lambda+s)^{-\alpha} X(s)$

(ii) $\mathscr{L}\left\{{ }_{0}^{C} D_{t}^{\alpha, \lambda} x(t)\right\}=(s+\lambda)^{\alpha} X(s)-\sum_{k=0}^{n-1}(s+\lambda)^{\alpha-k-1}$ $\left.\left[\left(\mathrm{d}^{k} / \mathrm{d} t^{k}\right)\left(e^{\lambda t} x(t)\right)\right]\right|_{t=0}$, where $X(s)=\mathscr{L}\{x(t)\}$ denotes the Laplace transform of $x(t)$

\section{Main Results}

In this section, tempered fractional comparison principles, some inequalities, and tempered Mittag-Leffler stability are derived.

3.1. Tempered Fractional Comparison Principles. In this section, we establish tempered fractional comparison principles.

Lemma 4. Assume that ${ }_{0}^{C} D_{t}^{\alpha, \lambda} x(t) \geq{ }_{0}^{C} D_{t}^{\alpha, \lambda} y(t), x(0)=y(0)$, $\alpha \in(0,1)$ and $\lambda \geq 0$, then $x(t) \geq y(t)$.

Proof. Following from ${ }_{0}^{C} D_{t}^{\alpha, \lambda} x(t) \geq{ }_{0}^{C} D_{t}^{\alpha, \lambda} y(t)$, there exists a function $m(t) \geq 0$ such that

$$
{ }_{0}^{C} D_{t}^{\alpha, \lambda} x(t)=m(t)+{ }_{0}^{C} D_{t}^{\alpha, \lambda} y(t)
$$

By Lemma 3, equation (5) yields

$$
\begin{aligned}
(s+\lambda)^{\alpha} X(s)-(s+\lambda)^{\alpha-1} x(0)= & M(s)+(s+\lambda)^{\alpha} Y(s) \\
& -(s+\lambda)^{\alpha-1} y(0) .
\end{aligned}
$$

According to $x(0)=y(0)$, we have

$$
(s+\lambda)^{\alpha} X(s)=M(s)+(s+\lambda)^{\alpha} Y(s),
$$

Thus,

$$
X(s)=Y(s)+(s+\lambda)^{-\alpha} M(s) .
$$

Taking the inverse Laplace transform on (8), solution of system (5) can be written as

$$
\begin{aligned}
x(t) & =y(t)+{ }_{0} I_{t}^{\alpha, \lambda} m(t) \\
& =y(t)+\frac{1}{\Gamma(\alpha)} \int_{0}^{t} e^{-\lambda(t-\tau)}(t-\tau)^{\alpha-1} m(\tau) \mathrm{d} \tau .
\end{aligned}
$$


According to $m(t) \geq 0$, therefore we obtain $x(t) \geq y(t)$.

Lemma 5. Assume that ${ }_{0} D_{t}^{\alpha, \lambda} x(t) \geq_{0} D_{t}^{\alpha, \lambda} y(t), x(0)=y(0)$, and $\alpha \in(0,1)$, then $x(t) \geq y(t)$.

Proof. From Lemma 1 and ${ }_{0} D_{t}^{\alpha, \lambda} x(t) \geq_{0} D_{t}^{\alpha, \lambda} y(t)$, we derive ${ }_{0}^{C} D_{t}^{\alpha, \lambda}(x(t))+\frac{e^{-\lambda t} t^{-\alpha}}{\Gamma(1-\alpha)} x(0) \geq{ }_{0}^{C} D_{t}^{\alpha, \lambda}(y(t))+\frac{e^{-\lambda t} t^{-\alpha}}{\Gamma(1-\alpha)} y(0)$.

That is ${ }_{0}^{C} D_{t}^{\alpha, \lambda}(x(t)) \geq{ }_{0}^{C} D_{t}^{\alpha, \lambda}(y(t))$. From Lemma 4 , we obtain $x(t) \geq y(t)$.

3.2. Some Inequalities. In this section, we construct some inequalities for tempered fractional derivatives or systems.

From Lemma 1, we could easily obtain the following lemma.

Lemma 6. The relationship between ${ }_{0}^{C} D_{t}^{\alpha, \lambda} x(t)$ and ${ }_{0} D_{t}^{\alpha, \lambda} x(t)$ is as follows:

$$
\left\{\begin{array}{l}
{ }_{0}^{C} D_{t}^{\alpha, \lambda} x(t) \leq{ }_{0} D_{t}^{\alpha, \lambda} x(t), \quad \text { if } x(0) \geq 0, \\
{ }_{0}^{C} D_{t}^{\alpha, \lambda} x(t) \geq_{0} D_{t}^{\alpha, \lambda} x(t), \quad \text { if } x(0) \leq 0,
\end{array}\right.
$$

where $\alpha \in(0,1)$.

Lemma 7. If $x(t) \in C^{1}([0,+\infty), \mathbb{R})$ is a continuously differentiable function, the following inequality holds:

$$
{ }_{0}^{C} D_{t}^{\alpha, \lambda}\left|x\left(t^{+}\right)\right| \leq \operatorname{sgn}\left(x(t){ }_{0}^{C} D_{t}^{\alpha, \lambda} x(t), \quad 0<\alpha<1, \lambda \geq 0,\right.
$$

where $x\left(t^{+}\right)=\lim _{\tau \longrightarrow t^{+}} x(t)$.

Proof. We take $y(t)=e^{\lambda t} x(t)$ into Theorem 2 in [22]

$$
{ }_{0}^{C} D_{t}^{\alpha}\left|y\left(t^{+}\right)\right| \leq \operatorname{sgn}(y(t))_{0}^{C} D_{t}^{\alpha} y(t), \quad 0<\alpha<1,
$$

for the Caputo fractional derivative. That is,

$$
{ }_{0}^{C} D_{t}^{\alpha}\left(e^{\lambda t}\left|x\left(t^{+}\right)\right|\right) \leq \operatorname{sgn}(x(t)){ }_{0}^{C} D_{t}^{\alpha}\left(e^{\lambda t} x(t)\right) .
$$

Multiplying both sides of equation (14) by $e^{-\lambda t}$, it gives $e^{-\lambda t C}{ }_{0}^{\alpha} D_{t}^{\alpha}\left(e^{\lambda t}\left|x\left(t^{+}\right)\right|\right) \leq \operatorname{sgn}(x(t)) e^{-\lambda t C}{ }_{0}^{\alpha} D_{t}^{\alpha}\left(e^{\lambda t} x(t)\right)$.

Using Definition 2, we obtain (12).

Consider the following tempered fractional system

$$
{ }_{t_{0}} D_{t}^{\alpha, \lambda} x(t)=f(t, x(t)), \quad 0<t<T,
$$

subjects to the proper initial conditions, where $\alpha \in(0,1)$, $\lambda \geq 0, D$ denotes either ${ }_{a}^{C} D_{t}^{\alpha, \lambda}$ or ${ }_{a} D_{t}^{\alpha, \lambda}, f:\left[t_{0},+\infty\right] \times \mathscr{D} \longrightarrow$ $\mathbb{R}^{n}$ is piecewise continuous in $t$ and locally Lipschitz in $x$, and $\mathscr{D} \subset \mathbb{R}^{n}$ is a domain contain the origin.

Theorem 1. For the real-valued continuous function $f(t, x)$ in (16), we have

$$
\left\|{ }_{0} I_{t}^{\alpha, \lambda} f(t, x)\right\| \leq_{0} I_{t}^{\alpha, \lambda}\|f(t, x)\|,
$$

where $\alpha>0, \lambda \geq 0$, and $\|\cdot\|$ denotes an arbitrary norm.

Proof. It follows from (1) that

$$
\begin{aligned}
\left\|{ }_{0} I_{t}^{\alpha, \lambda} f(t, x(t))\right\| & =\left\|\frac{1}{\Gamma(\alpha)} \int_{0}^{t} e^{-\lambda(t-s)}(t-s)^{\alpha-1} f(s, x(s)) \mathrm{d} s\right\| \\
& \leq \frac{1}{\Gamma(\alpha)} \int_{0}^{t} e^{-\lambda(t-s)}(t-s)^{\alpha-1}\|f(s, x(s))\| \mathrm{d} s \\
& ={ }_{0} I_{t}^{\alpha, \lambda}\|f(t, x(t))\| .
\end{aligned}
$$

Theorem 2. If $x=0$ is an equilibrium point of system (16) with ${ }_{t_{0}} D_{t}^{\alpha, \lambda}={ }_{0}^{C} D_{t}^{\alpha, \lambda}$ and $f(t, x)$ is Lipschitz on $x$ with Lipschitz constant $l$ and piecewise continuous with respect to $t$; then, we have

$$
\|x(t)\| \leq\|x(0)\| e^{-\lambda t} E_{\alpha}\left(l t^{\alpha}\right) .
$$

Proof. By applying the tempered fractional integral operator ${ }_{0} I_{t}^{\alpha, \lambda}$ to system (16), it follows from Lemma 2 and Lipschitz condition in $f(t, x)$ that

$$
\begin{aligned}
\|x(t)\|-e^{-\lambda t}\|x(0)\| & \leq\left\|x(t)-e^{-\lambda t} x(0)\right\| \\
& =\left\|I_{0} I_{t}^{\alpha, \lambda}\left[{ }_{0}^{C} D_{t}^{\alpha, \lambda} x(t)\right]\right\|=\left\|_{0} I_{t}^{\alpha, \lambda} f(t, x(t))\right\| \\
& \leq{ }_{0} I_{t}^{\alpha, \lambda}\|f(t, x(t))\| \leq l_{0} I_{t}^{\alpha, \lambda}\|x(t)\| .
\end{aligned}
$$

There exists a function $M(t) \geq 0$ such that

$$
\|x(t)\|-e^{-\lambda t}\|x(0)\|=l_{0} I_{t}^{\alpha, \lambda}\|x(t)\|-M(t) .
$$

Combining with Lemma 3 and Laplace transform to (21), we obtain

$$
\|x(s)\|=\frac{(s+\lambda)^{\alpha-1}\|x(0)\|-(s+\lambda)^{\alpha} M(s)}{(s+\lambda)^{\alpha}-l},
$$

where $\|x(s)\|=\mathscr{L}\{\|x(t)\|\}$. Taking the inverse Laplace transform to (22) gives

$$
\|x(t)\|=\|x(0)\| e^{-\lambda t} E_{\alpha}\left(l t^{\alpha}\right)-M(t) *\left[e^{-\lambda t} t^{-1} E_{\alpha, 0}\left(l t^{\alpha}\right)\right],
$$

where $*$ denotes the convolution operator. Obviously, $e^{-\lambda t} t^{-1} E_{\alpha, 0}\left(l t^{\alpha}\right) \geq 0, \quad$ then inequality $\|x(t)\| \leq\|x(0)\| e^{-\lambda t} E_{\alpha}\left(l t^{\alpha}\right)$ is obtained.

3.3. Tempered Mittag-Leffler Stability. In this section, some sufficient conditions are established for the tempered Mittag-Leffler stability of system (16).

Definition 4. If and only if $f(t, \bar{x})={ }_{t_{0}} D_{t}^{\alpha, \lambda} \bar{x}$, then $\bar{x} \in \mathbb{R}^{n}$ is an equilibrium point of tempered fractional system (16). 
Definition 5. Assume $\bar{x}=0$ is an equilibrium point of system (16), the solution of (16) is said to be tempered Mittag-Leffler stable if

$$
\|x(t)\| \leq\left[m\left(x\left(t_{0}\right)\right) e^{-\lambda t} E_{\alpha}\left(-l\left(t-t_{0}\right)^{\alpha}\right)\right]^{b},
$$

where $\alpha>0, \lambda \geq 0, l>0, b>0$, and $m(0)=0, m(x) \geq 0$ satisfies locally Lipschitz condition.

Remark 1. Tempered Mittag-Leffler stability is a generalization of Mittag-Leffler stability. When $\lambda=0$, tempered Mittag-Leffler stability can be reduced to Mittag-Leffler stability $[26,27]$.

Remark 2. Both Mittag-Leffler stability and tempered Mittag-Leffler stability imply asymptotic stability, that is, $\|x(t)\| \longrightarrow 0$ as $t \longrightarrow \infty$.

Theorem 3. Assume $x=0$ be an equilibrium point for (16) and domain $\mathscr{D} \subset \mathbb{R}^{n}$ contains the origin. Let $V(t, x(t)):[0,+\infty) \times \mathscr{D} \longrightarrow \mathbb{R}$ be a continuously differentiable function and locally Lipschitz with respect to $x$, such that

$$
\begin{gathered}
\alpha_{1}\|x(t)\|^{a} \leq V(t, x(t)) \leq \alpha_{2}\|x(t)\|^{a b}, \\
{ }_{0}^{C} D_{t}^{\beta, \lambda} V(t, x(t)) \leq-\alpha_{3}\|x(t)\|^{a b},
\end{gathered}
$$

where $t \geq 0, x \in \mathscr{D}, \beta \in(0,1)$, and $\alpha_{1}, \alpha_{2}, \alpha_{3}, a$, and $b$ are given positive constants, then $x=0$ is tempered Mittag-Leffler stable. If the assumptions hold globally on $\mathbb{R}^{n}$, then $x=0$ is globally tempered Mittag-Leffler stable.

Proof. It follows from equations (25) and (26) that

$$
{ }_{0}^{C} D_{t}^{\beta, \lambda} V(t, x(t)) \leq-\frac{\alpha_{3}}{\alpha_{2}} V(t, x(t)) \text {. }
$$

There exists a function $M(t) \geq 0$ such that

$$
{ }_{0}^{C} D_{t}^{\beta, \lambda} V(t, x(t))+M(t)=-\frac{\alpha_{3}}{\alpha_{2}} V(t, x(t)) .
$$

Taking the Laplace transform to (28) gives

$$
(s+\lambda)^{\beta} V(s)-(s+\lambda)^{\beta-1} V(0)+M(s)=-\frac{\alpha_{3}}{\alpha_{2}} V(s) \text {, }
$$

where nonnegative constant $V(0)=V(0, x(0))$ and $V(s)=\mathscr{L}\{V(t, x(t))\}$. We rewrite this in the form

$$
V(s)=\frac{V(0)(s+\lambda)^{\beta-1}-M(s)}{(s+\lambda)^{\beta}+\left(\alpha_{3} / \alpha_{2}\right)} .
$$

Applying the inverse Laplace transform to (30), unique solution of (28) is

$$
V(t)=V(0) e^{-\lambda t} E_{\beta}\left(-\frac{\alpha_{3}}{\alpha_{2}} t^{\beta}\right)-M(t) *\left[e^{-\lambda t} t^{\beta-1} E_{\beta, \beta}\left(-\frac{\alpha_{3}}{\alpha_{2}} t^{\beta}\right)\right] .
$$

Because $t^{\beta-1}$ and $E_{\beta, \beta}\left(-\left(\alpha_{3} / \alpha_{2}\right) t^{\beta}\right)$ are nonnegative functions, we obtain

$$
V(t) \leq V(0) e^{-\lambda t} E_{\beta}\left(-\frac{\alpha_{3}}{\alpha_{2}} t^{\beta}\right)
$$

Substituting (32) into (25) satisfies

$$
\|x(t)\| \leq\left[\frac{V(0)}{\alpha_{1}} e^{-\lambda t} E_{\beta}\left(-\frac{\alpha_{3}}{\alpha_{2}} t^{\beta}\right)\right]^{1 / a},
$$

and $x(0)=0$ if and only if $\left(V(0) / \alpha_{1}\right) e^{-\lambda t}=0$.

Because $V(t, x)$ is local Lipschitz condition with respect to $x$ and $x(0)=0$ if and only if $V(0, x(0))=0$, then $\left(V(0, x(0)) / \alpha_{1}\right) e^{-\lambda t}$ satisfies local Lipschitz condition about $x(0)$. So, system (16) is tempered Mittag-Leffler stable.

Theorem 4. Assume all conditions in Theorem 3 are satisfied except replacing ${ }_{0}^{C} D_{t}^{\beta, \lambda}$ by ${ }_{0} D_{t}^{\beta, \lambda}$, then we have

$$
\|x(t)\| \leq\left[\frac{V(0)}{\alpha_{1}} e^{-\lambda t} E_{\beta}\left(-\frac{\alpha_{3}}{\alpha_{2}} t^{\beta}\right)\right]^{1 / a} .
$$

Proof. It follows from Lemma 6 and $V(t, x) \geq 0$ that

$$
{ }_{0}^{C} D_{t}^{\beta, \lambda} V(t, x) \leq{ }_{0} D_{t}^{\beta, \lambda} V(t, x) \leq-\alpha_{3}\|x(t)\|^{a b} .
$$

A similar proof method in Theorem 3 shows result (35).

Theorem 5. For the tempered fractional system (10), where ${ }_{0} D_{t}^{\alpha, \lambda}={ }_{0} D_{t}^{\alpha, \lambda}, f(t, x)$ is Lipschitz on $x$ with constant $l>0$, and $f(t, 0)=0$, if there exists a Lyapunov candidate $V(t, x)$ yielding

$$
\begin{aligned}
& \alpha_{1}\|x(t)\|^{a} \leq V(t, x(t)) \leq \alpha_{2}\|x(t)\| \\
& \dot{V}(t, x(t)) \leq-\alpha_{3}\|x(t)\|
\end{aligned}
$$

where $\alpha_{3}>\lambda \alpha_{2}, \alpha_{1}, \alpha_{2}, \alpha_{3}, a$ are given positive constants and $\dot{V}(t, x(t))=(d V(t, x) / d t)$, then

$$
\|x(t)\| \leq\left[\frac{V(0)}{\alpha_{1}} e^{-\lambda t} E_{1-\alpha}\left(-\frac{\alpha_{3}-\lambda \alpha_{2}}{\alpha_{2}} t^{1-\alpha}\right)\right]^{1 / a} .
$$

Proof. From (1) and (2), we find that tempered fractional derivative on $V(t, x)$ can be represented in the form

$$
\begin{aligned}
{ }_{0}^{C} D_{t}^{1-\alpha, \lambda} V(t, x)= & \frac{e^{-\lambda t}}{\Gamma(\alpha)} \int_{0}^{t} \frac{1}{(t-s)^{1-\alpha}} \frac{\mathrm{d}\left(e^{\lambda s} V(s, x(s))\right)}{\mathrm{d} s} \mathrm{~d} s \\
= & \frac{e^{-\lambda t}}{\Gamma(\alpha)} \int_{0}^{t} \frac{1}{(t-s)^{1-\alpha}}\left(\lambda e^{\lambda s} V(s, x(s))\right. \\
& \left.+e^{\lambda s} \dot{V}(s, x(s))\right) \mathrm{d} s \\
= & \lambda \frac{e^{-\lambda t}}{\Gamma(\alpha)} \int_{0}^{t} \frac{e^{\lambda s} V(s, x(s))}{(t-s)^{1-\alpha}} \mathrm{d} s+\frac{e^{-\lambda t}}{\Gamma(\alpha)} \int_{0}^{t} \\
& \frac{e^{\lambda s} \dot{V}(s, x(s))}{(t-s)^{1-\alpha} \mathrm{d} s} \\
= & \lambda_{0} I_{t}^{\alpha, \lambda} V(t, x(t))+{ }_{0} I_{t}^{\alpha, \lambda} \dot{V}(t, x(t)) .
\end{aligned}
$$


Using (36) and (37) and Lipschitz condition on $f(t, x)$, we obtain

$$
\begin{aligned}
{ }_{0}^{C} D_{t}^{1-\alpha, \lambda} V(t, x) & \leq \lambda \alpha_{2} I_{t}^{\alpha, \lambda}\|x(t)\|-\alpha_{30} I_{t}^{\alpha, \lambda} \| x(t) \\
& =-\left(\alpha_{3}-\lambda \alpha_{2}\right)_{0} I_{t}^{\alpha, \lambda}\|x(t)\| \| \\
& \leq-\left(\alpha_{3}-\lambda \alpha_{2}\right) l^{-1}{ }_{0} I_{t}^{\alpha, \lambda}\|f(t, x(t))\| .
\end{aligned}
$$

We can use Lemmas 7 and 2 to write

$$
\begin{aligned}
{ }_{0}^{C} D_{t}^{1-\alpha, \lambda} V(t, x) & \leq-\left(\alpha_{3}-\lambda \alpha_{2}\right) l^{-1}\left\|_{0} I_{t}^{\alpha, \lambda} f(t, x(t))\right\| \\
& =-\left(\alpha_{3}-\lambda \alpha_{2}\right) l^{-1}\|x(t)\|,
\end{aligned}
$$

where $\left.{ }_{0} D_{t}^{\alpha-1}\left(e^{\lambda t} u(t)\right)\right|_{t=0}=0$. By the same proof in Theorem 3 , conclusion (38) holds.

\section{Applications}

In this section, we will give three examples to demonstrate theoretical analysis. The Adams-Bashforth-Moulton method [37] is employed for solving tempered fractional differential equations in the simulations.

Example 1. Consider the tempered fractional Riemann-Liouville system:

$$
{ }_{0} D_{t}^{\alpha, \lambda} x(t)=-|x(t)|, \quad 0<\alpha<1, \lambda \geq 0,
$$

where $x(0)>0$. The Lyapunov function candidate is chosen as $V(t, x)=|x|$. From Lemma 1, we obtain

$$
{ }_{0}^{C} D_{t}^{\alpha, \lambda} V={ }_{0}^{C} D_{t}^{\alpha, \lambda}|x| \leq{ }_{0} D_{t}^{\alpha, \lambda}|x|=-|V| .
$$

By Theorem 3, we have

$$
|x(t)| \leq|x(0)| e^{-\lambda t} E_{\alpha}\left(-t^{\alpha}\right) .
$$

Then, $x=0$ is tempered Mittag-Leffler stable. To verify the result, we choose parameters as $\alpha=0.95$ and $x(0)=4$ and the tempered parameters as $\lambda=2,4,6,8$, respectively. The time evolution of the system states (42) is shown in Figure 1. It is presented that the solution of system (42) converges to the equilibrium point $x=0$. The larger the tempered parameter $\lambda$ is, the faster the convergence speed becomes.

Example 2. Consider the tempered fractional Caputo Hopfield neural networks:

$$
{ }_{0}^{C} D_{t}^{\alpha, \lambda} x_{i}(t)=-a_{i} x_{i}(t)+\sum_{j=1}^{n} b_{i j} f_{j}\left(x_{j}(t)\right)+I_{i},
$$

where $0<\alpha<1, \lambda \geq 0, i=1,2, \ldots, n$ and $n$ is number of units. $x_{i}(t)$ is the $i$ th state, $f_{j}$ is the $j$ th activation function, $b_{i j}$ is constant connection weight of the $j$ th neuron on the $i$ th neuron, $a_{i}>0$ denotes the resting rate when the $i$ th neuron disconnected, and $I_{i}$ is the external inputs. Under the conditions

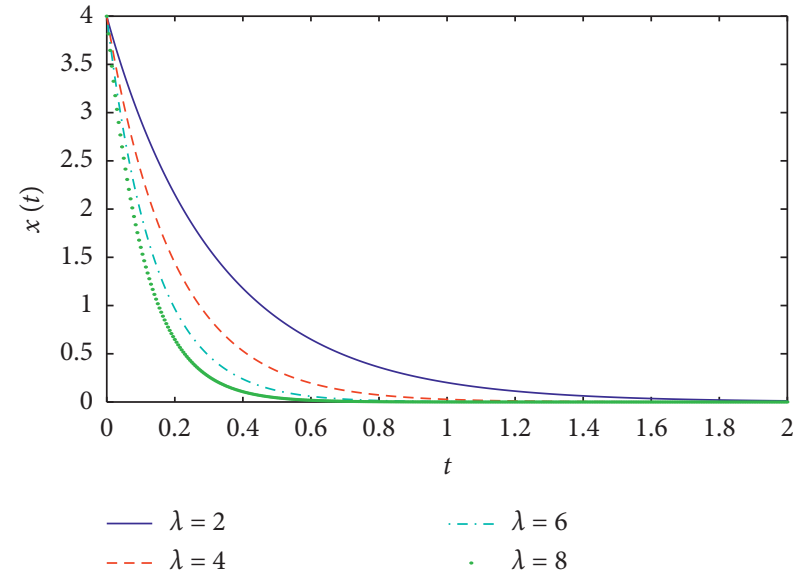

FIgURE 1: Time evolution of system states $x(t)$ with different tempered parameters $\lambda$ in Example 1.

$$
\begin{aligned}
\left|f_{j}(x)\right| \leq l_{j}|x|, \quad l_{j}>0, j=1,2, \ldots, n, & \\
c_{i} & =a_{i}-\sum_{j=1}^{n}\left|b_{j i}\right| l_{i}>0, \quad i=1,2, \ldots, n,
\end{aligned}
$$

system (45) is globally tempered Mittag-Leffler stable.

Let $x(t)=\left(x_{1}, x_{2}, \ldots, x_{n}\right)^{T}$ be any solution of system (45). We choose Lyapunov function as follows:

$$
V(t, x(t))=\|x(t)\|=\sum_{i=1}^{n}\left|x_{i}(t)\right|
$$

By inequalities (46) and (47) and Lemma 7, tempered fractional Caputo derivative on $V(t, x(t))$ can be written as

$$
\begin{aligned}
{ }_{0}^{C} D_{t}^{\alpha, \lambda} V(t, x(t)) & =\sum_{i=1}^{n}{ }_{0}^{C} D_{t}^{\alpha, \lambda}\left|x_{i}(t)\right| \leq \sum_{i=1}^{n} \operatorname{sgn}\left(x_{i}(t)\right){ }_{0}^{C} D_{t}^{\alpha, \lambda} x_{i}(t) \\
& \leq-a_{i} \sum_{i=1}^{n}\left|x_{i}(t)\right|+\sum_{i=1}^{n} \sum_{j=1}^{n} l_{j}\left|b_{i j}\right|\left|x_{j}(t)\right| \\
& =\sum_{i=1}^{n}\left[-a_{i}+\sum_{j=1}^{n} l_{i}\left|b_{j i}\right|\right]\left|x_{i}(t)\right| \\
& \leq-c\|x(t)\|,
\end{aligned}
$$

where $c=\min \left\{c_{1} \cdot c_{2}, \ldots, c_{n}\right\}$. From (49) and Theorem 3, system (45) is globally tempered Mittag-Leffler stable.

To illustrate the effectiveness of Example 2, in system (45), we let $\quad x=\left(x_{1}, x_{2}, x_{3}\right)^{T}, \alpha=0.98$, $x_{1}(0)=5, x_{2}(0)=-3, x_{3}(0)=3, f_{j}\left(x_{j}\right)=\tanh \left(x_{j}\right)$, and $c_{j}=6$ for $j=1,2,3$ and

$$
A=\left(a_{i j}\right)_{3 \times 3}=\left[\begin{array}{ccc}
2 & -1.2 & 0 \\
1.8 & 1.71 & 1.15 \\
-4.75 & 0 & 1.1
\end{array}\right] .
$$

It is obvious that condition (47) is satisfied. Let tempered parameters $\lambda=0,2,4,6$, respectively. As shown in Figure 2, 


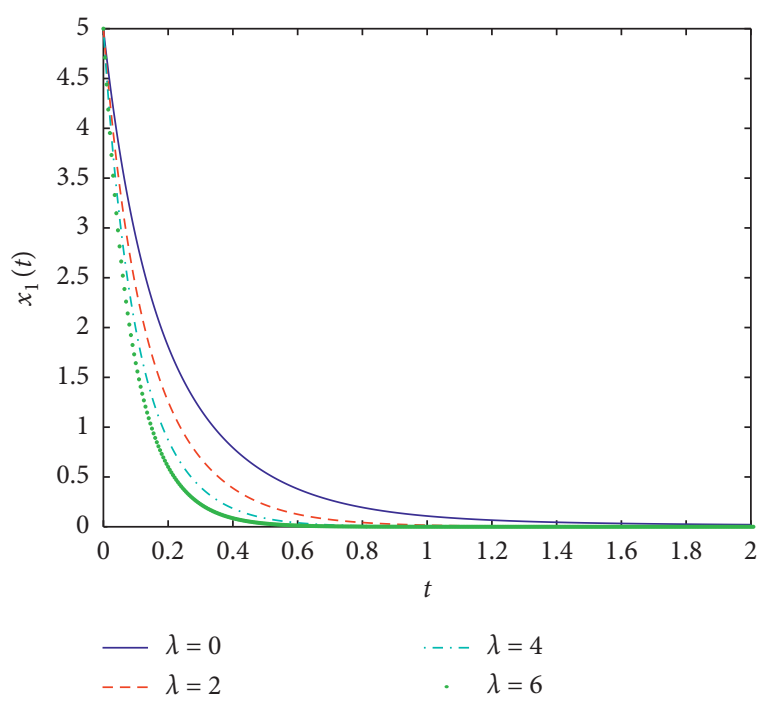

(a)

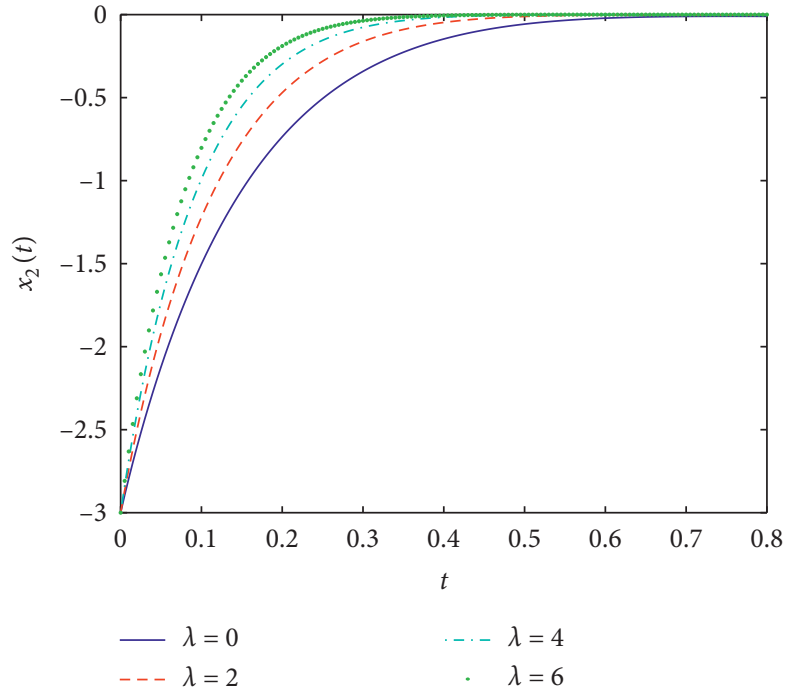

(b)

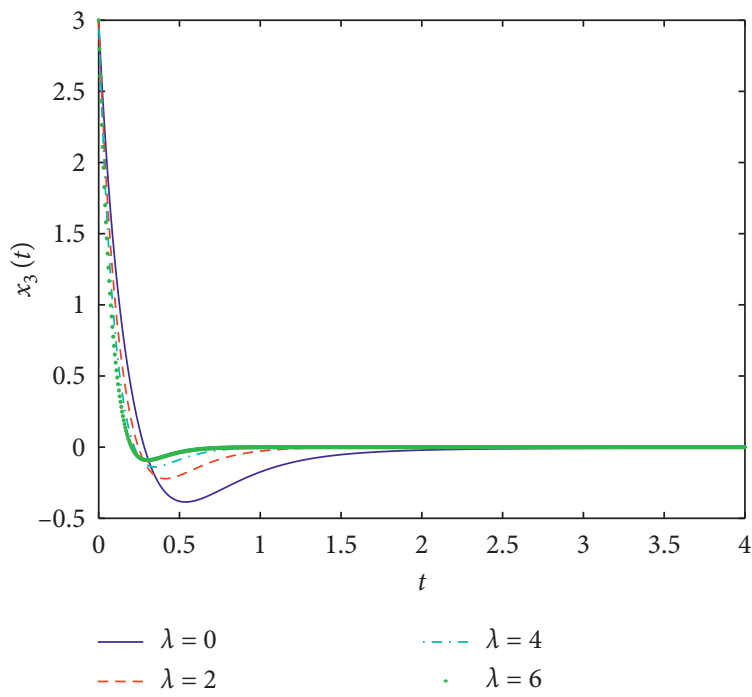

(c)

FIGURE 2: Time evolution of system states $x(t)$ with different tempered parameters $\lambda$ in Example 2.

the equilibrium point $x=0$ is tempered Mittag-Leffler stable and the solution of system (45) converges to $x=0$.

Example 3. Consider the following tempered fractional system:

$$
\begin{aligned}
& { }_{0}^{C} D_{t}^{\alpha, \lambda} x_{1}=-2 x_{1}+\frac{\sin \left(x_{3}\right)}{1+t^{2}} x_{1}, \\
& { }_{0}^{C} D_{t}^{\alpha, \lambda} x_{2}=-2 x_{2}+\cos \left(x_{1}\right) x_{2}, \\
& { }_{0}^{C} D_{t}^{\alpha, \lambda} x_{3}=x_{3},
\end{aligned}
$$

where $0<\alpha<1, \lambda \geq 0$ and $x(t)=\left(x_{1}(t), x_{2}(t), x_{3}(t)\right) \in \mathbb{R}^{3}$.

Let the Lyapunov function $V(t, x)=\left|x_{1}\right|+\left|x_{2}\right|$. By Lemma 7 , we obtain

$$
\begin{aligned}
{ }_{0}^{C} D_{t}^{\alpha, \lambda} V(t, x) \leq & \operatorname{sgn}\left(x_{1}(t){ }_{0}^{C} D_{t}^{\alpha, \lambda} x_{1}(t)+\operatorname{sgn}\left(x_{2}(t)\right)_{0}^{C} D_{t}^{\alpha, \lambda} x_{2}(t)\right. \\
= & \operatorname{sgn}\left(x_{1}(t)\right)\left(-2 x_{1}(t)+\frac{\sin \left(x_{3}(t)\right)}{1+t^{2}} x_{1}(t)\right) \\
& +\operatorname{sgn}\left(x_{2}(t)\right)\left(-2 x_{2}(t)+\cos \left(x_{1}(t)\right) x_{2}(t)\right) \\
\leq & -2\left|x_{1}(t)\right|+\frac{\sin \left(x_{3}(t)\right)}{1+t^{2}}\left|x_{1}(t)\right|-2\left|x_{2}(t)\right| \\
& +\cos \left(x_{1}(t)\right)\left|x_{2}(t)\right| \\
\leq & -\left(\left|x_{1}(t)\right|+\left|x_{2}(t)\right|\right) .
\end{aligned}
$$




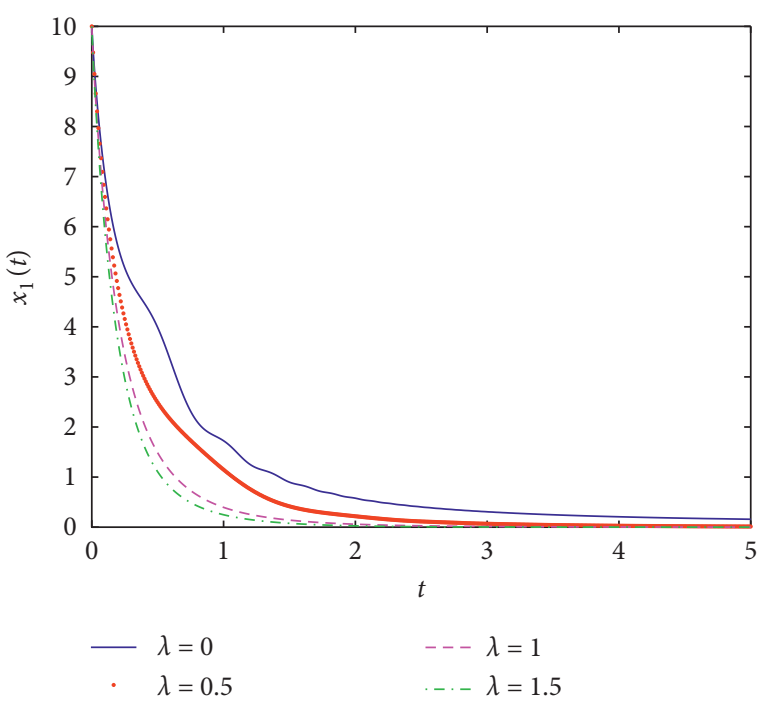

(a)

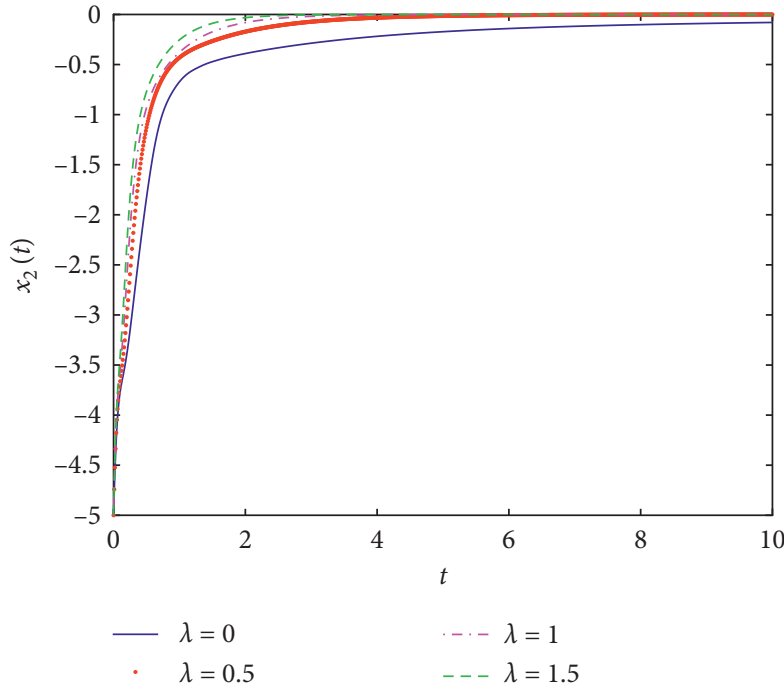

(b)

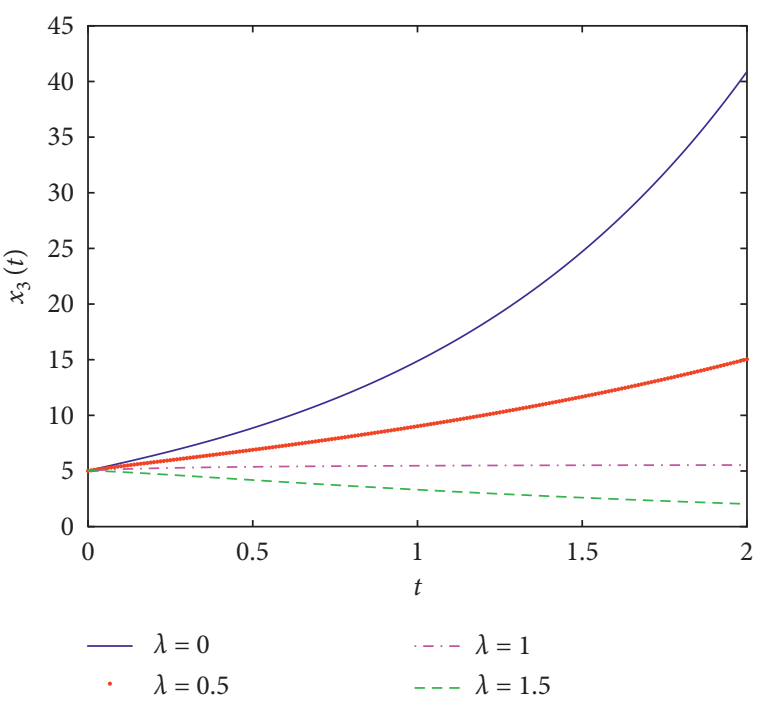

(c)

FIgURE 3: Time evolution of system states $x(t)$ with different tempered parameters $\lambda$ in Example 3.

Then, the conditions of Theorem 3 are satisfied. Hence, $x=0$ is globally tempered Mittag-Leffler stable with respect to $\left(x_{1}, x_{2}\right)$. Take $\alpha=0.9, x_{1}(0)=10, x_{2}(0)=-5$, and $x_{3}(0)=$ 5. The numerical simulation is shown as Figure 3 with different tempered parameters $\lambda=0,0.5,1,1.5$. It is obvious $x_{1}(t)$ and $x_{2}(t)$ converge to 0 . When tempered parameter of the system increase, the part solution of system converges faster.

\section{Conclusions}

In this paper, we present some stability results for the tempered fractional systems. Based on the Laplace transform, we obtain the comparison principle for the tempered fractional systems. Some theorems about tempered Mittag-Leffler stability are derived, which enrich the knowledge of the system theory and the tempered fractional calculus and are helpful in characterizing the tempered fractional system models. Furthermore, we will study stability of tempered fractional systems with time-varying delays in future work.

\section{Data Availability}

The authors affirm that all data necessary for confirming the conclusions of the article are present in the article.

\section{Conflicts of Interest}

The authors declare that there are no conflicts of interest regarding the publication of this paper.

\section{Acknowledgments}

This work was supported by the introduction of talent, the Northwest University for Nationalities, special Funds for Talents (nos. xbmuyjrc201916, xbmuyjrc201632), the 
Humanities and Social Sciences Planning Projects of the Ministry of Education of P. R. China under Grant (no. 19YJAZH010), and Fundamental Research Funds for the Central Universities (no. 31920180119).

\section{References}

[1] A. Einstein, "Über die von der molekularkinetischen theorie der wärme geforderte bewegung von in ruhenden flüssigkeiten suspendierten teilchen," Annalen der Physik, vol. 322, no. 8, pp. 549-560, 1905.

[2] J. Klafter and I. M. Sokolov, "Anomalous diffusion spreads its wings," Physics World, vol. 18, no. 8, pp. 29-32, 2005.

[3] F. Sabzikar, M. M. Meerschaert, and J. Chen, "Tempered fractional calculus," Journal of Computational Physics, vol. 293, pp. 14-28, 2015.

[4] R. N. Mantegna and H. E. Stanley, "Stochastic process with ultraslow convergence to a Gaussian: the truncated lévy flight," Physical Review Letters, vol. 73, no. 22, pp. 2946-2949, 1994.

[5] I. Koponen, "Analytic approach to the problem of convergence of truncated Lévy flights towards the Gaussian stochastic process," Physical Review E, vol. 52, no. 1, pp. 1197-1199, 1995.

[6] Á. Cartea and D. del-Castillo-Negrete, "Fluid limit of the continuous-time random walk with general Lévy jump distribution functions," Physical Review E, vol. 76, no. 4, Article ID 041105, 2007.

[7] P. Carr, H. Geman, D. B. Madan, and M. Yor, "The fine structure of asset returns: an empirical investigation," The Journal of Business, vol. 75, no. 2, pp. 305-332, 2002.

[8] P. Carr, H. Geman, D. B. Madan, and M. Yor, "Stochastic volatility for Lévy processes," Mathematical Finance, vol. 13, no. 3, pp. 345-382, 2003.

[9] Á. Cartea and D. del-Castillo-Negrete, "Fractional diffusion models of option prices in markets with jumps," Physica A: Statistical Mechanics and its Applications, vol. 374, no. 2, pp. 749-763, 2007.

[10] O. E. Barndorff-Nielsen, "Processes of normal inverse Gaussian type," Finance and Stochastics, vol. 2, no. 1, pp. 41-68, 1997.

[11] M. M. Meerschaert, Y. Zhang, and B. Baeumer, "Tempered anomalous diffusions in heterogeneous systems," Geophysical Research Letters, vol. 35, no. 17, 2008.

[12] Y. Zhang and M. M. Meerschaert, "Gaussian setting time for solute transport in fluvial systems," Water Resources Research, vol. 47 , no. $8,2011$.

[13] M. M. Meerschaert, F. Sabzikar, M. S. Phanikumar, and A. Zeleke, "Tempered fractional time series model for turbulence in geophysical flows," Journal of Statistical Mechanics: Theory and Experiment, vol. 2014, no. 9, p. 9023, 2014.

[14] M. M. Meerschaert and F. Sabzikar, "Tempered fractional Brownian motion," Statistics \& Probability Letters, vol. 83, no. 10, pp. 2269-2275, 2013.

[15] C. Li and F. Zhang, "A survey on the stability of fractional differential equations," The European Physical Journal Special Topics, vol. 193, no. 1, pp. 27-47, 2011.

[16] C. Jiang, F. Zhang, and T. Li, "Synchronization and antisynchronization of $\mathrm{N}$-coupled fractional-order complex chaotic systems with ring connection," Mathematical Methods in the Applied Sciences, vol. 41, no. 7, pp. 2625-2638, 2018.

[17] C. Jiang, A. Zada, M. Tamer Senel, and T. Li, "Synchronization of bidirectional $\mathrm{N}$-coupled fractional-order chaotic systems with ringconnection based on antisymmetric structure," Advances in Difference Equations, vol. 2019, no. 1, p. 456, 2019.

[18] S. Huang and Q. Tian, "Marcinkiewicz estimates for solution to fractional elliptic Laplacian equation," Computers \& Mathematics with Applications, vol. 78, no. 5, pp. 1732-1738, 2019.

[19] M. Hong, W. Sun, S. Liu, and T. Xuan, "Coherence analysis and Laplacian energy of recursive trees with controlled initial states," Frontiers of Information Technology \& Electronic Engineering, 2019.

[20] W. Sun, M. Sun, J. Guan, and Q. Jia, "Robustness of coherence in noisy scale-free networks and applications to identification of influential spreaders," IEEE Transactions on Circuits and Systems II: Express Briefs, 2020.

[21] X. Gao and J. Yu, "Chaos in the fractional order periodically forced complex Duffing's oscillators," Chaos, Solitons and Fractals, vol. 24, no. 4, pp. 1097-1104, 2005.

[22] S. Zhang, Y. Yu, and H. Wang, "Mittag-Leffler stability of fractional-order Hopfield neural networks," Nonlinear Analysis: Hybrid Systems, vol. 16, pp. 104-121, 2015.

[23] S. Yang, J. Yu, C. Hu, and H. Jiang, "Quasi-projective synchronization of fractional-order complex-valued recurrent neural networks," Neural Networks, vol. 104, pp. 104-113, 2018.

[24] W. Ma, C. Li, Y. Wu, and Y. Wu, "Synchronization of fractional fuzzy cellular neural networks with interactions," Chaos: An Interdisciplinary Journal of Nonlinear Science, vol. 27, no. 10, Article ID 103106, 2017.

[25] E. Ahmed, A. M. A. El-Sayed, and H. A. A. El-Saka, "Equilibrium points, stability and numerical solutions of fractionalorder predator-prey and rabies models," Journal of Mathematical Analysis and Applications, vol. 325, no. 1, pp. 542-553, 2007.

[26] Y. Li, Y. Chen, and I. Podlubny, "Mittag-Leffler stability of fractional order nonlinear dynamic systems," Automatica, vol. 45, no. 8, pp. 1965-1969, 2009.

[27] Y. Li, Y. Chen, and I. Podlubny, "Stability of fractional-order nonlinear dynamic systems: Lyapunov direct method and tempered Mittag-Leffler stability," Computers \& Mathematics with Applications, vol. 59, no. 5, pp. 1810-1821, 2010.

[28] P. Liu, Z. Zeng, and J. Wang, "Multiple Mittag-Leffler stability of fractional-order recurrent neural networks," IEEE Transactions on Systems, Man, and Cybernetics: Systems, vol. 47, no. 8, pp. 2279-2288, 2017.

[29] W. Chen, H. Dai, Y. Song, and Z. Zhang, "Convex Lyapunov functions for stability analysis of fractional order systems," IET Control Theory \& Applications, vol. 11, no. 7, pp. 10701074, 2017.

[30] X. Yang, C. Li, T. Huang, and Q. Song, "Mittag-Leffler stability analysis of nonlinear fractional-order systems with impulses," Applied Mathematics and Computation, vol. 293, pp. 416-422, 2017.

[31] W. Deng, B. Li, W. Tian, and P. Zhang, "Boundary problems for the fractional and tempered fractional operators," Multiscale Modeling \& Simulation, vol. 16, no. 1, pp. 125-149, 2018.

[32] W. Deng and Z. Zhang, "Variational formulation and efficient implementation for solving the tempered fractional problems," Numerical Methods for Partial Differential Equations, vol. 34, no. 4, pp. 1224-1257, 2018.

[33] W. Ma, C. Li, and J. Deng, "Synchronization in tempered fractional complex networks via auxiliary system approach," Complexity, vol. 2019, Article ID 6071412, 12 pages, 2019. 
[34] A. Fernandez and C. Ustaoğlu, "On some analytic properties of tempered fractional calculus," Journal of Computational and Applied Mathematics, vol. 366, Article ID 112400, 2020.

[35] Y. Yan, W. Deng, and D. Nie, "A finite-difference approximation for the one-and two-dimensional tempered fractional Laplacian," Communications on Applied Mathematics and Computation, vol. 2, no. 1, pp. 129-145, 2020.

[36] C. Li, W. Deng, and L. Zhao, "Well-posedness and numerical algorithm for the tempered fractional ordinary differential equations," American Institute of Mathematical Science, vol. 24, no. 4, pp. 1989-2015, 2019.

[37] J. Deng, L. Zhao, and Y. Wu, "Fast predictor-corrector approach for the tempered fractional differential equations," Numerical Algorithms, vol. 74, no. 3, pp. 717-754, 2017. 